\title{
RESEARCH
}

Open Access

\section{CircLRP6 contributes to prostate cancer growth and metastasis by binding to miR- 330-5p to up-regulate NRBP1}

Linghui Qin, Xiaosong Sun, Fei Zhou and Cheng Liu*

\begin{abstract}
Background: Circular RNA low-density lipoprotein receptor-related protein 6 (circLRP6) is considered as an oncogene in many types of cancers. However, the function and mechanisms of circLRP6 in prostate cancer (PCa) tumorigenesis remain largely undefined.

Methods: Quantitative real-time polymerase chain reaction (qRT-PCR) and western blot assays were conducted to assess the expression of circLRP6, microRNA (miR)-330-5p, and nuclear receptor binding protein 1 (NRBP1). Cell counting kit-8 (CCK-8), colony formation, 5-ethynyl-2'-deoxyuridine (EDU) incorporation, flow cytometry, transwell, wound healing, and western blot assays were performed to detect cell proliferation, apoptosis, and metastasis in vitro. Subcutaneous tumor growth was observed in nude mice to investigate the role of circLRP6 in vivo. The targeting relationship between miR-330-5p and NRBP1 or circLRP6 was verified using dual-luciferase reporter, pulldown, and RNA immunoprecipitation (RIP) assays. Immunohistochemistry was employed to test relative protein expression.
\end{abstract}

Results: CircLRP6 was highly expressed in PCa tissues and cells, knockdown of circLRP6 impaired PCa cell growth and metastasis in vitro by affecting cell proliferation, apoptosis, invasion, migration, and epithelial-mesenchymal transition (EMT). Mechanistic studies showed that circLRP6 could competitively bind with miR-330-5p to prevent the degradation of its target gene NRBP1. Rescue assay suggested that miR-330-5p inhibition reversed the inhibitory effects of circLRP6 knockdown on PCa cell growth and metastasis. Moreover, overexpression of miR-330-5p suppressed PCa progression via NRBP1. Notably, tumor formation assay indicated that circLRP6 silencing impeded tumor growth and EMT in vivo.

Conclusion: Our findings demonstrated that circLRP6 promoted PCa tumorigenesis and metastasis through miR330-5p/NRBP1 axis, suggesting a potential therapeutic target for PCa.

Keywords: circLRP6, MiR-330-5p, NRBP1, Prostate cancer, Metastasis, Growth

\footnotetext{
*Correspondence: ivncdd@163.com

Department of Urology, Xiangyang Central Hospital, Affiliated Hospital of Hubei University of Arts and Science, No. 136, Jingzhou Street, Xiangyang 441021, Hubei, China
}

C C The Author(s). 2021 Open Access This article is licensed under a Creative Commons Attribution 4.0 International License, which permits use, sharing, adaptation, distribution and reproduction in any medium or format, as long as you give appropriate credit to the original author(s) and the source, provide a link to the Creative Commons licence, and indicate if changes were made. The images or other third party material in this article are included in the article's Creative Commons licence, unless indicated otherwise in a credit line to the material. If material is not included in the article's Creative Commons licence and your intended use is not permitted by statutory regulation or exceeds the permitted use, you will need to obtain permission directly from the copyright holder. To view a copy of this licence, visit http://creativecommons.org/licenses/by/4.0/ The Creative Commons Public Domain Dedication waiver (http://creativecommons.org/publicdomain/zero/1.0/) applies to the data made available in this article, unless otherwise stated in a credit line to the data. 


\section{Background}

Prostate cancer $(\mathrm{PCa})$ is the most common malignancy in men through the world, with an estimated 1.6 million new cases and 366,000 deaths annually [1, 2]. Despite the high long-term survival in localized $\mathrm{PCa}$ and the effectivity of androgen deprivation therapy in the control of metastatic $\mathrm{PCa}$, the majority of patients still develop castration-resistant $\mathrm{PCa}$ (CRPCa) [3-5]. Thus, further investigations on the pathogenesis and identifications on novel bio-labelling therapy in $\mathrm{PCa}$ are of great significance.

Currently, accumulating evidence has suggested that noncoding RNAs, including circular RNAs (circRNAs), microRNAs (miRNAs/miRs), and long noncoding RNAs (lncRNAs), impact a variety of biological processes, such as cell proliferation, apoptosis, and metastasis [6]. Unlike linear RNAs, circRNAs possess covalently closed loop structures that lack the 3 ' and 5' ends, and are highly stable relative to their linear counterparts in vivo due to the resistance to the degradation by RNA exonuclease [7, 8]. Besides that, most of circRNAs are highly conserved across species and exhibit development- or tissue-specific expression pattern [9]. Importantly, recent reports have proved the involvement of circRNAs in cancer progression by acting as carcinogens or tumor suppressors $[10,11]$. Thus, circRNAs may be promising therapeutic targets for cancers. In $\mathrm{PCa}$, some circRNAs, such as circFoxo3 [12], circUCK2 [13], and circ_0044516 [14], were also found to be aberrantly expressed and functioned as a critical type of endogenous RNAs to regulate cancer cell survival, invasion, migration and therapeutic resistance, thus affecting PCa tumorigenesis. CircRNAs low-density lipoprotein receptor-related protein 6 (circLRP6) (ID: hsa_circ_0000378) is derived from exons 2 of the LRP6 gene. A previous study suggested that highly expressed circLRP6 was related to the worse overall survival in osteosarcoma, and circLRP6 promoted osteosarcoma tumorigenesis by downregulating KLF2 and APC expression level [15]. Xue et al. revealed that circLRP6 enhanced arsenite-evoked malignant transformation in human keratinocyte cells by inducing cell epithelial-mesenchymal transition (EMT) via miR-455/ZEB1 axis [16]. Besides, circLRP6 was also found to upregulate Myc expression level by targeting miR-182 to promote cell proliferation and invasion in esophageal squamous cell cancer [17]. Therefore, circLRP6 may function as an oncogene in cancer progression.

In this study, we speculated that circLRP6 might also serve as a carcinogen in $\mathrm{PCa}$ progression. Here, the action of circLRP6 in PCa tumorigenesis in vitro and in vivo was investigated. Besides that, how
circLRP6 affected the tumorigenesis of PCa was further explored.

\section{Materials and methods}

\section{Clinical specimens}

Seventy-one tissue samples, including 19 normal prostate tissues and 52 PCa tissues, were collected from PCa patients admitted to Xiangyang Central Hospital, and then stored at $-80{ }^{\circ} \mathrm{C}$ until further used. All specimen diagnoses were confirmed by pathological examinations after surgery. The study equipped with written informed consent from each enrolled individual.

\section{In situ hybridization (ISH)}

Paraffin-embedded tissues from PCa patients were collected. CircLRP6-positive expression was examined using a specific digoxigenin (DIG)-labeled circLRP6 probe designed by Geneseed (Guangzhou, China). After being dewaxed in xylene and rehydrated through gradient alcohol, the tissue microarray (TMA) was digested using proteinase $\mathrm{K}$ and hybridized with the specific circLRP6 probe overnight at $4{ }^{\circ} \mathrm{C}$, followed by incubation with anti-DIG-AP (Roche, Basel, Switzerland) overnight $4{ }^{\circ} \mathrm{C}$. After staining with nitro blue tetrazolium (NBT)/5bromo-4-chloro-3-indolyl phosphate (BCIP) (Roche), the staining results were observed.

\section{Cell culture}

Human RWPE-1 cells, human PCa cell lines (PC3 and DU145), and $293 \mathrm{~T}$ cell lines were purchased from Cedarlane (Burlington, NC, USA). All cells were cultivated in RPMI-1640 medium (Sigma, St. Louis, MO, USA) plus penicillin/streptomycin $(100 \mathrm{U} / \mathrm{mL}$, Gibco, Rockville, MD, USA) and 10\% fetal bovine serum (FBS, Gibco) at $37{ }^{\circ} \mathrm{C}$ with $5 \% \mathrm{CO}_{2}$.

\section{Quantitative real-time polymerase chain reaction (qRT- PCR)}

The isolation of total RNA was conducted using the TRIzol (Life Technologies, Waltham, MA, USA). Total RNA $(2 \mu \mathrm{g})$ was incubated for $30 \mathrm{~min}$ at $37^{\circ} \mathrm{C}$ with or without $3 \mathrm{U} / \mu \mathrm{g}$ of RNase R (Epicentre Technologies, Madison, WI, USA) to verify the circular characteristics of circRNA. Reverse transcription was performed using the PrimeScript RT Reagent Kit (Takara, Dalian, China) under recommended condition. Then $\mathrm{SYBR}^{\circ}$ Select Master Mix (Takara) was employed to perform qRT-PCR. U6 or glyceraldehyde-3-phosphate dehydrogenase (GAPDH) was used as the normalization control and the expression levels of molecules were examined using the $2^{-\Delta \Delta C t}$ method. The primer sequences for qRT-PCR were listed: circLRP6: F 5'-GAGTTGGATCAACCCAGAGC-3', R 5'-TCCTCCAAGCCTCCAACTAC-3'; 
Nuclear Receptor Binding Protein 1 (NRBP1): F 5'GAGGTGAATCAACGGAATGTACC-3'， R 5'-CTTG TAGTTCTTGCGTTCAGAGA-3'; LRP6: F 5'-TTTATGCAAACAGACGGGACTT-3', R 5'-GCCTCCAACTACAATCGTAGC-3';

GAPDH: F 5'-CCCACATGGCCTCCAAGGAGTA-3', R 5'-GTGTACATGGCAACTGTGAGGAGG-3'; miR-330-5p: F 5'-GGGACACAGGGCCAGAGAC-3', R 5'-GGGACACAGGGCCAGAGAC-3';

U6: F 5'-CTCGCTTCGGCAGCACA-3', R 5'-AACG CTTCACGAATTTGCGT-3'.

\section{Cell transfection}

The siRNA duplexes targeting circLRP6 (si-circLRP6\#1, si-circLRP6\#2 and si-circLRP6\#3) and scrambled siRNA (si-NC), pcDNA3.1 NRBP1 overexpressing plasmid (pcDNA-NRBP1) and negative control (pcDNA), miR330-5p mimic, miR-330-5p inhibitor, mimic-control (mimic NC), and inhibitor-control (inhibitor NC) were obtained from GenePharma (Shanghai, China). The PC3 and DU145 cells grown to $60-70 \%$ confluence were transfected with miRNA mimics, miRNA inhibitors, plasmids siRNAs, or respective controls using Lipofectamine 2000 (Invitrogen, San Diego, CA, USA).

\section{Cell proliferation assays}

Cell proliferation was determined by cell counting Kit-8 (CCK-8), colony formation, and 5-Ethynyl-2'-Deoxyuridine (EDU) incorporation assays. For CCK-8 assay, transfected PC3 and DU145 cells were placed into 96well plates with $3 \times 10^{3}$ per well. At 24,48 , or $72 \mathrm{~h}$ incubation, each well was added with $10 \mu \mathrm{L}$ of CCK-8 reagent (Beyotime, Shanghai, China) and incubated for another $1 \mathrm{~h}$. The absorbance in each well was measured using a microplate reader at $450 \mathrm{~nm}$ (Bio-Rad, Hercules, CA, USA). For colony formation assay, PC3 and DU145 following assigned transfection were plated at 6-well plates (500 cells/well). After culture for 14 days, cell colonies were fixed with ethanol (Beyotime), and the number of colonies was imaged and counted after crystal violet staining. For the EDU incorporation assay, an EDU incorporation assay kit (RiboBio, Guangzhou, China) was used. Transfected PC3 and DU145 cells were incubated with $50 \mathrm{mM}$ EDU for $2 \mathrm{~h}$. Then, cells were fixed with $4 \%$ paraformaldehyde (Beyotime) and stained with Apollo Dye Solution for proliferating cells. Subsequently, the nuclei were stained with DAPI. The images were obtained employing a fluorescence microscope, and stained cells were analyzed using ImageJ software (National Institutes of Health, Sacaton, AZ, USA).

\section{Cell cycle and apoptosis analysis}

Transfected PC3 and DU145 cells were trypsinized and rinsed with pre-cold phosphate buffer solution (PBS).
Cell supernatant was discarded after centrifugation, and then cells were fixed with $70 \%$ cold ethanol for $1 \mathrm{~h}$. After washing with $\mathrm{PBS}$, fixed cells were stained with propidium iodide (PI) (BD Biosciences, Franklin Lakes, NJ, USA), and the FACScan flow cytometer (BD Biosciences) was applied to determine cell cycle distribution.

For cell apoptosis analysis, transfected PC3 and DU145 cells were resuspended in $500 \mu \mathrm{L} 1 \times$ Annexin binding buffer to obtain a concentration of $5 \times 10^{5} / \mathrm{mL}$, followed by staining with Annexin V-fluorescein isothiocyanate (FITC) (BD Biosciences) $(5 \mu \mathrm{L})$ and propidium iodide (PI) $(10 \mu \mathrm{L})$. Cell apoptosis was detected using the FACScan flow cytometry (BD Biosciences).

\section{Cell invasion and migration analysis}

Cell invasion was analyzed by transwell chambers (pore size $8 \mu \mathrm{m}$; Costar, Cambridge, MA, USA) with matrigelcoated filters (BD Biosciences). Transfected PC3 and DU145 cells $\left(2 \times 10^{5}\right.$ cells $)$ were plated in the top chamber. The lower chamber contained $600 \mu \mathrm{L}$ medium with $10 \%$ FBS was used as a chemoattractant. The cells were incubated at $37{ }^{\circ} \mathrm{C}$ with $5 \% \mathrm{CO}_{2}$ for $24 \mathrm{~h}$. The number of invaded cells in five random fields of the lower surface was counted after crystal violet staining.

Cell migration was analyzed using wound healing assay. After transfection, PC3 and DU145 cells were incubated in a 6-well plate containing RPMI-1640 plus $10 \%$ FBS. When cells grown to a fully confluent monolayer, cells were scratched using a $200-\mu \mathrm{L}$ pipette tip and then grown in the serum-free medium. At 0 and $24 \mathrm{~h}$, the width of wounds was detected and captured.

\section{Western blot}

Total protein was extracted with RIPA buffer and the protein concentration was quantified using a BCA protein assay kit (Com Win Biotech, Qingdao, China). A total of $50 \mu \mathrm{g}$ protein was separated by $10 \%$ SDS-polyacrylamide gel electrophoresis, and then transferred onto the Clear Blot membrane-p (ATTO, Tokyo, Japan). The blots were incubated with anti-E-Cadherin (ab1416), anti-Vimentin (ab8978), anti-GAPDH (ab181602) (all 1:1000; Abcam, Shanghai, China), and anti-NRBP1 (Cat\# H00029959M01, 1:1000, Abnova, Taipei, Taiwan) overnight at $4{ }^{\circ} \mathrm{C}$ and then incubated with HRP-labeled specific secondary antibody for $1 \mathrm{~h}$ at room temperature. Protein blots were visualized with an enhanced chemoluminescence kit (KeyGen) and scanned using Image software based on the mixture of both intensity and band thickness.

\section{Dual-luciferase reporter assay}

The bindings between miR-330-5p and NRBP1 or circLRP6 was predicted by starBase3.0 (http://starbase. sysu.edu.cn/) or Circinteractome database (https:// circinteractome.nia.nih.gov/), respectively [18]. Wild- 
type plasmids circLRP6 wt and NRBP1 wt, and mutanttype plasmids circLRP6 mut and NRBP1 mut were inserted into the pGL3 promoter vector (GenePharma, Shanghai, China). 293T cells were seeded into 24-well plates and co-transfected with $100 \mathrm{ng}$ of reporter plasmids, $20 \mathrm{ng}$ of pRL-TK Renilla Luciferase vector and 50 $\mathrm{nM}$ miRNAs mimics/negative control using Lipofectamine 2000 (Invitrogen). A dual-luciferase reporter assay kit (Promega, Madison, WI, USA) was employed to analyze the luciferase activity.

\section{RNA immunoprecipitation (RIP) assay}

PC3 and DU145 cells were firstly lysed in RIP lysis buffer (Millipore, Billerica, MA, USA) and then incubated with RNA magnetic beads (Millipore) conjugated with Anti-Ago2 (ab32381, Abcam) or anti-IgG. At last, the immunoprecipitated RNAs were eluted, purified and subjected to qRT-PCR.

\section{Pull-down assay}

Biotinylated miR-330-5p (Bio-miR-330-5p) and its negative control (Bio-NC) were generated by Sangon (Shanghai). PC3 and DU145 cells were lysed and the lysate was incubated with streptavidin-coated magnetic beads containing Bio-miR-330-5p or Bio-NC. Following being eluted, the levels of circLRP6 and NRBP1 were detected using qRT-PCR.

\section{Xenograft experiments}

Male BABL/c nude mice (5 weeks old) were obtained from Charles River Laboratory (Beijing, China) and randomly divided into two groups ( $N=6$ per group). PC3 cells $\left(2 \times 10^{6}\right.$ cells $/ 0.2 \mathrm{~mL}$ PBS $)$ stably infected lentivirus-mediated sh-NC or sh-circLRP6 (GeneCopoeia, Rockville, MD, USA) were subcutaneously injected into the right flank of nude mice, respectively, and mice were maintained in Specific Pathogen Free conditions. Tumor size was detected using a caliper every week and tumor volume was calculated with the equation: volume $=\left(\right.$ length $\times$ width $\left.^{2}\right) / 2$. At day 35, tumors were removed and weighted for further molecular analysis with qRT-PCR and western blot, respectively.

\section{IHC assay}

The tumors collected from in vivo tumor formation assay were fixed in formalin and then embedded in paraffin. Paraffin-embedded tumors were deparaffinized in xylene and rehydrated through graded alcohol to water. Then tumor sections were treated with 3\% hydrogen peroxide to block endogenous peroxidase activity. After incubation with $5 \%$ bovine serum albumin (BSA), the sections were incubated with anti-Ki-67 (ab15580, Abcam), anti-NRBP1 (Cat\# H00029959-M01, Abnova) (all 1:100) overnight at $4{ }^{\circ} \mathrm{C}$, secondary antibodies for 1 h at $37{ }^{\circ} \mathrm{C}$ and HRP-labeled streptavidin solution for 10 min. Diaminobenzidine (DAB) was used as chromogen.

\section{Statistical analysis}

Data from three repeated experiments were presented as mean \pm standard deviation. The data were statistically analyzed using Student's $t$ test (two-sided) or analysis of variance followed by Tukey's post hoc analysis. $P<0.05$ was considered statistically significant.

\section{Results}

CircLRP6 is highly expressed in PCa tissues and cells

According to the public database (GSE113153), multiple circRNAs were found to be differentially expressed in the high-grade and low-grade PCa tissues, among them, circLRP6 expression was found to be significantly higher than other circRNAs (Fig. 1A). Moreover, the expression of circLRP6 was detected in clinical tissues. As expected, circLRP6 was highly expressed in PCa tissues, especially in specimens of patients with high-grade $\mathrm{PCa}$, relative to the normal tissues (Fig. 1B). Moreover, ISH analysis suggested that circLRP6 in PCa tissues was elevated compared with normal tissues (Fig. 1C). In parallel, the expression of circLRP6 was apparently elevated in PCa cells (PC3 and DU145) compared with the RWPE-1 cells (Fig. 1D). CircLRP6 (ID: has_circ_0000378) is derived from exons 2 of the LRP6 gene, whose spliced mature sequence length is $394 \mathrm{bp}$ (Fig. 1E). Next, we investigated the stability of circLRP6 in PCa cells (PC3 and DU145). PC3 and DU145 cells were treated with or without RNase R, which has a $3^{\prime}-5^{\prime}$ exoribonuclease activity, then it was found that circLRP6 was markedly resistant to RNase $\mathrm{R}$ degradation relative to the linear LRP6 mRNA (Fig. 1F), demonstrating that circLRP6 was more stable than LRP6.

\section{Knockdown of circLRP6 suppresses PCa cell growth and metastasis}

To determine the biological function of circLRP6 in PCa cells in vitro, short interference siRNAs against circLRP6 (si-circLRP6\#1, si-circLRP6\#2, si-circLRP6\#3) were designed and synthesized to knock down circLRP6 in PC3 and DU145 cells, and the knockdown efficiency was confirmed by qRT-PCR (Fig. 2A).The si-circLRP6\#2 and si-circLRP6\#3 were selected for subsequent functional analysis. After circLRP6 silencing, the proliferation (Fig. 2B), colony-formation abilities (Fig. 2C), and DNA synthesis activities (Fig. 2D) of PC3 and DU145 cells were markedly decreased, evidenced by the CCK-8, colony formation, and EDU incorporation assays. The results of flow cytometry suggested that silencing of circLRP6 led to an arrest of PC3 and DU145 cells at G0/G1 phase, accompanying by the decreased percentage of cells in $\mathrm{S}$ phase (Fig. 2E). In addition, transwell and wound healing 


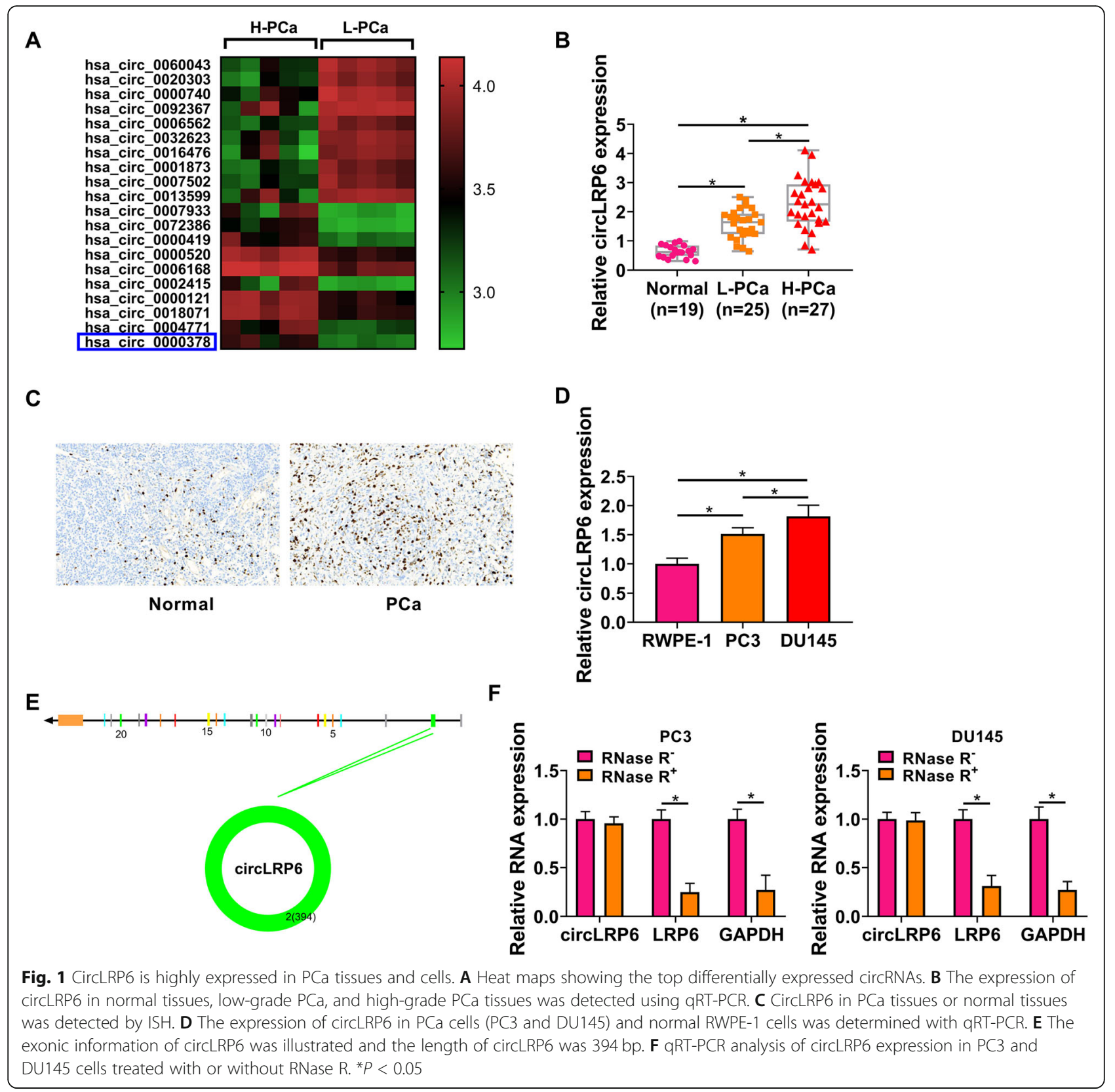

assays showed that circLRP6 knockdown suppressed the invasion (Fig. 2F) and migration (Fig. 2G) activities of PC3 and DU145 cells. Besides that, the apoptosis of PC3 and DU145 cells was found to be significantly increased by the knockdown of circLRP6 (Fig. 2H). Moreover, the effects of circLRP6 on EMT, one of major mechanisms for cancer metastasis, were assessed. Western blot analysis showed that E-cadherin was increased while Vimentin was decreased in circLRP6-downregulated PC3 and DU145 cells (Fig. 2I). Taken together, knockdown of circLRP6 suppressed PCa cell growth and metastasis in vitro.
MiR-330-5p is a target of circLRP6 in PCa cells

The potential targeted miRNAs interacted by circLRP6 were then investigated. Bioinformatics analysis (Circinteractome database) revealed that 12 miRNAs were predicted to contain a putative binding site in circLRP6. Through the search of previous studies and results of qRT-PCR, miR-1247, miR-153, miR-198, miR-326, miR515-5p, miR-543, and miR-330-5p were revealed to be associated with $\mathrm{PCa}$, among them, the expression levels of miR-198, miR-326, miR-515-5p, miR-543, and miR330-5p were decreased in PCa, importantly, miR-330-5p 


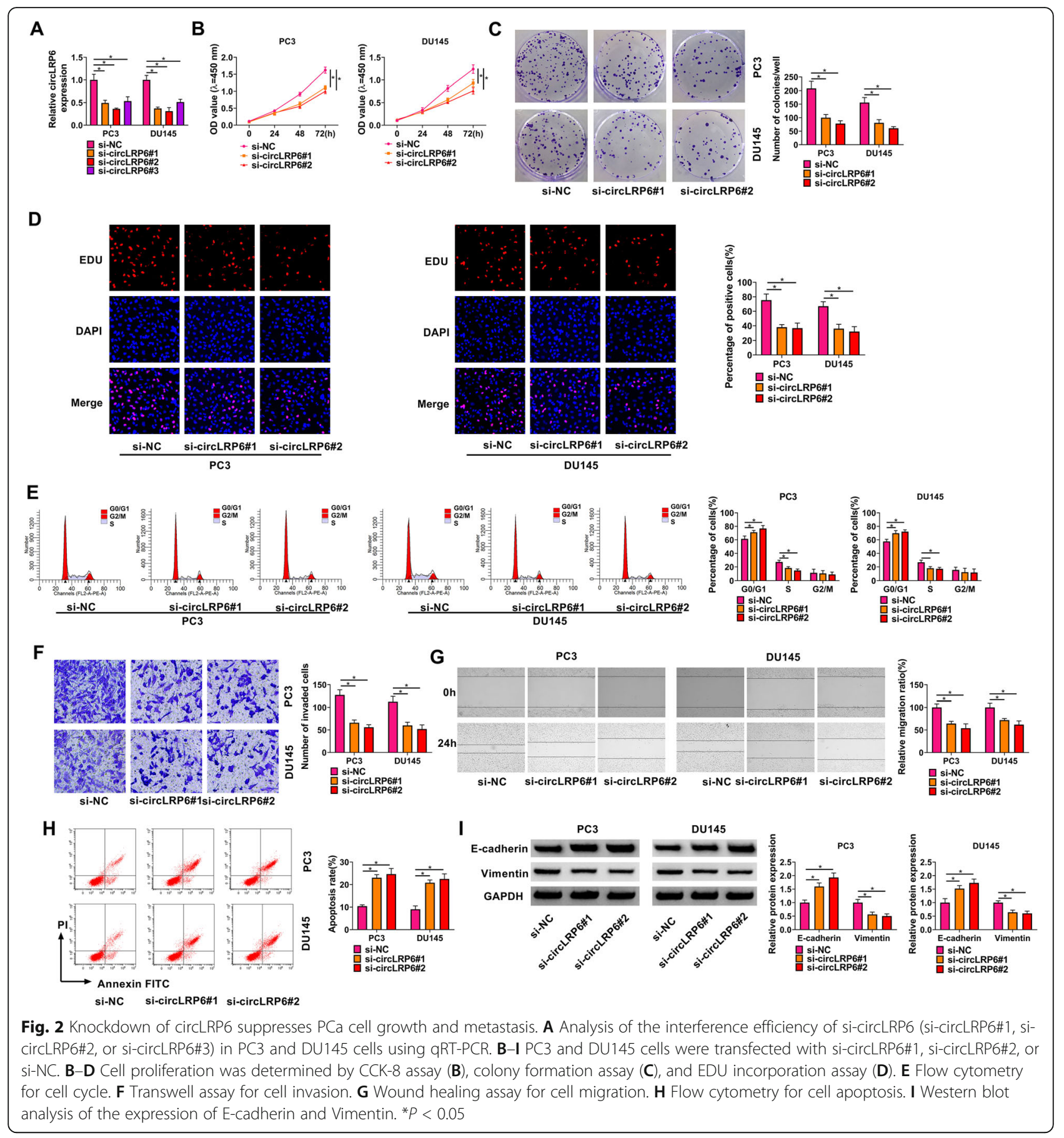

expression was significantly affected by circLRP6 knockdown (Fig. S2A-C). Thus, miR-330-5p was selected for subsequent analysis. The putative binding site between circLRP6 and miR-330-5p was shown in Fig. 3A. Then, dual-luciferase reporter assay was carried out. The results suggested that miR-330-5p mimic significantly reduced the luciferase activity of wild-type circLRP6 reporter but not the mutated one in $293 \mathrm{~T}$ cells (Fig. 3B).
Further RIP assay exhibited that the Ago2 antibody could pull down both endogenous circLRP6 and miR330-5p in PC3 and DU145 cells (Fig. 3C). Moreover, pull-down assay implied that circLRP6 was significantly enriched by Bio-miR-330-5p group compared with BioNC group (Fig. 3D). All these results confirmed the direct interaction between circLRP6 and miR-330-5p. After that, the expression profile of miR-330-5p was 
A
circLRP6 wt 5'-CAAGAGUUGGAUCAACCCAGAGC-3' | | | | | | miR-330-5p 3' CGGAUUCUGUGUCCGGGUCUCU 5' circLRP6 mut 5'-CAAGAGUUGGAUCAAGGGUCUCC-3'

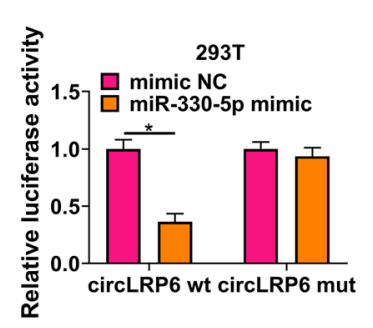

C
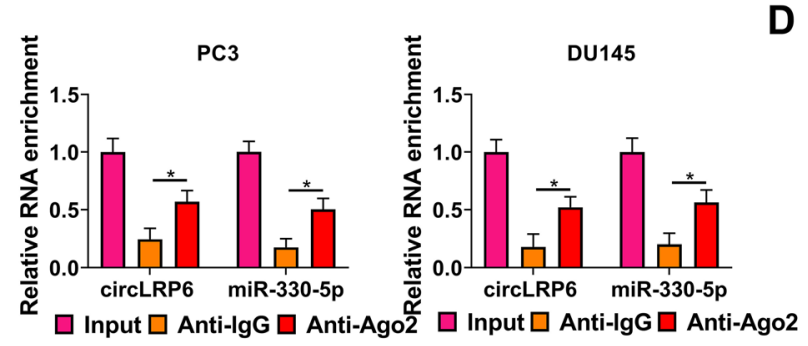

E

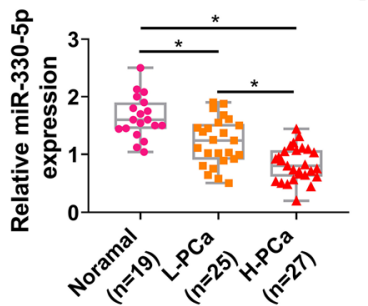

$\mathbf{F}$

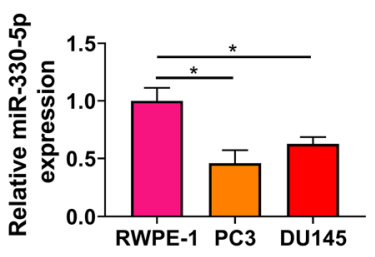

G
D

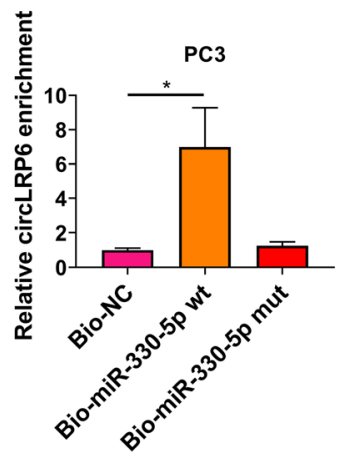

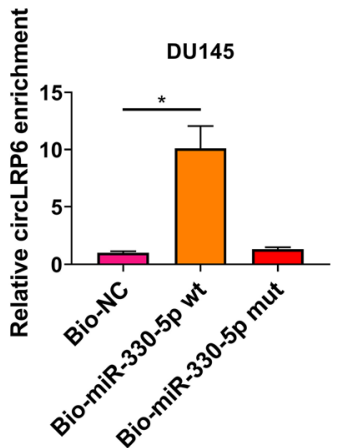

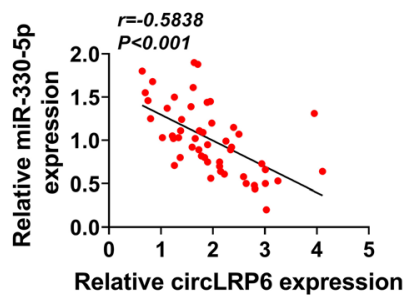

Fig. 3 MiR-330-5p is a target of circLRP6 in PCa cells. A The putative binding site between circLRP6 and miR-330-5p. B Dual-luciferase reporter assay for the relative luciferase activities of the wild and mutated circLRP6 reporter after miR-330-5p upregulation in 293T cells. C RIP assay for the examination of circLRP6 and miR-330-5p enrichment in immunoprecipitation complexes in PC3 and DU145 cells. D Biotin-labeled pull-down assay indicating the interaction between circLRP6 and miR-330-5p in PC3 and DU145 cells. E The expression of miR-330-5p in normal tissues, lowgrade PCa and high-grade PCa tissues was detected using qRT-PCR. $\mathbf{F}$ The expression of miR-330-5p in PCa cells (PC3 and DU145) and normal RWPE-1 cells was determined with qRT-PCR. G MiR-330-5p expression was negatively correlated with circLRP6 in PCa tissues. ${ }^{*} P<0.05$

investigated. As shown in Fig. 3E, miR-330-5p was found to be decreased in $\mathrm{PCa}$ tissues, especially in high-grade PCa tissues. Similarly, its expression was also decreased in PCa cells compared with the normal RWPE-1 cells (Fig. 3F). Moreover, a negative correlation between miR330-5p and circLRP6 expression was observed in PCa tissues (Fig. 3G). In all, these results verified that miR330-5p was an inhibitory target of circLRP6 in PCa cells.

\section{Knockdown of circLRP6 suppresses PCa cell growth and metastasis via targeting miR-330-5p}

Next, we examined whether miR-330-5p mediated the action of circLRP6 on PCa cells. qRT-PCR analysis suggested that the introduction of miR-330-5p inhibitor remarkably reduced the expression level of miR-330-5p in PC3 and DU145 cells compared with the negative control (Fig. 4A). Then miR-330-5p inhibitors were transfected into circLRP6-decreased PC3 and DU145 cells, as expected, miR-330-5p inhibitors reversed circLRP6 knockdown-induced elevation of miR-330-5p in cells (Fig. 4B). Thereafter, rescue assay was performed. It was proved that miR-330-5p inhibition attenuated circLRP6 knockdown-evoked suppression of cell proliferation (Fig. 4C), colony-formation abilities (Fig. 4D), and DNA synthesis activities (Fig. 4E) in PC3 and DU145 cells. The arrest of cell cycle (Fig. 4F) and reduction of cell apoptosis (Fig. 4G) induced by circLRP6 knockdown were partially abolished by the inhibition of miR-330-5p. Furthermore, transfection of miR-330-5p inhibitors reversed circLRP6 knockdown-mediated cell metastasis inhibition, reflected by the increase of the invaded and migrated PC3 and DU145 cells (Fig. 4H, I), as well as the progression of EMT in PC3 and DU145 cells (Fig. 4J). Additionally, we also determined the effects of 


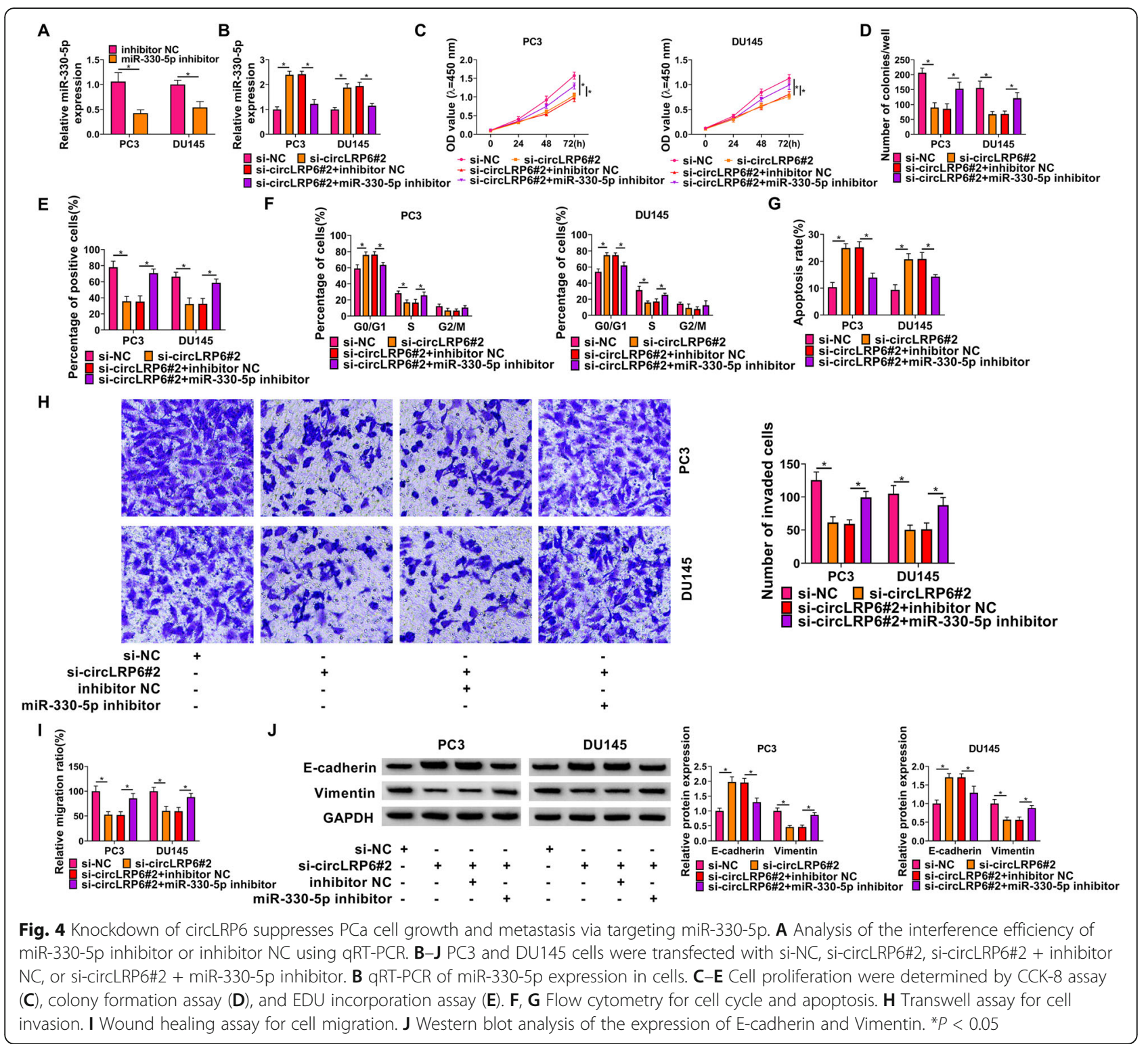

circLRP6/miR-330-5p axis on normal RWPE-1 cells. As shown in Fig. S1A, miR-330-5p inhibitor also reduced circLRP6 knockdown-induced increase of miR-330-5p expression in RWPE-1 cells. Then it was found that the circLRP6/miR-330-5p axis had no effects on RWPE-1 cells (Fig. S1B-D). Altogether, circLRP6 affected PCa cell growth and metastasis via regulating miR-330-5p.

\section{MiR-330-5p directly targets NRBP1 in PCa cells}

Based on the results of bioinformatics analysis, numerous genes were predicted to have the binding site of miR-330$5 \mathrm{p}$, among which, IGF1R, MYO6, SMAD3, CCND1, and NRBP1 were selected due to their high expression in PCa according the previous researches. Thereafter, western blot analysis showed that NRBP1 expression was overtly reduced by miR-330-5p up-regulation (Fig. S2C, D). The putative binding site of NRBP1 and miR-330-5p was exhibited in Fig. 5A. The results of dual-luciferase reporter assay showed that miR-330-5p mimics reduced the luciferase activity in the wild-type rather than mutated type of NRBP1 in 293T cells (Fig. 5B). RIP assay further exhibited that the levels of miR-330-5p and NRBP1 were visibly enriched in anti-Ago2 group compared with that in antiIgG group in PC3 and DU145 cells (Fig. 5C). Pull-down assay indicated that the enrichment level of NRBP1 in the Bio-miR-330-5p group was significantly higher than that in the Bio-NC group (Fig. 5D), further confirming the binding between miR-330-5p and NRBP1. Thereafter, 


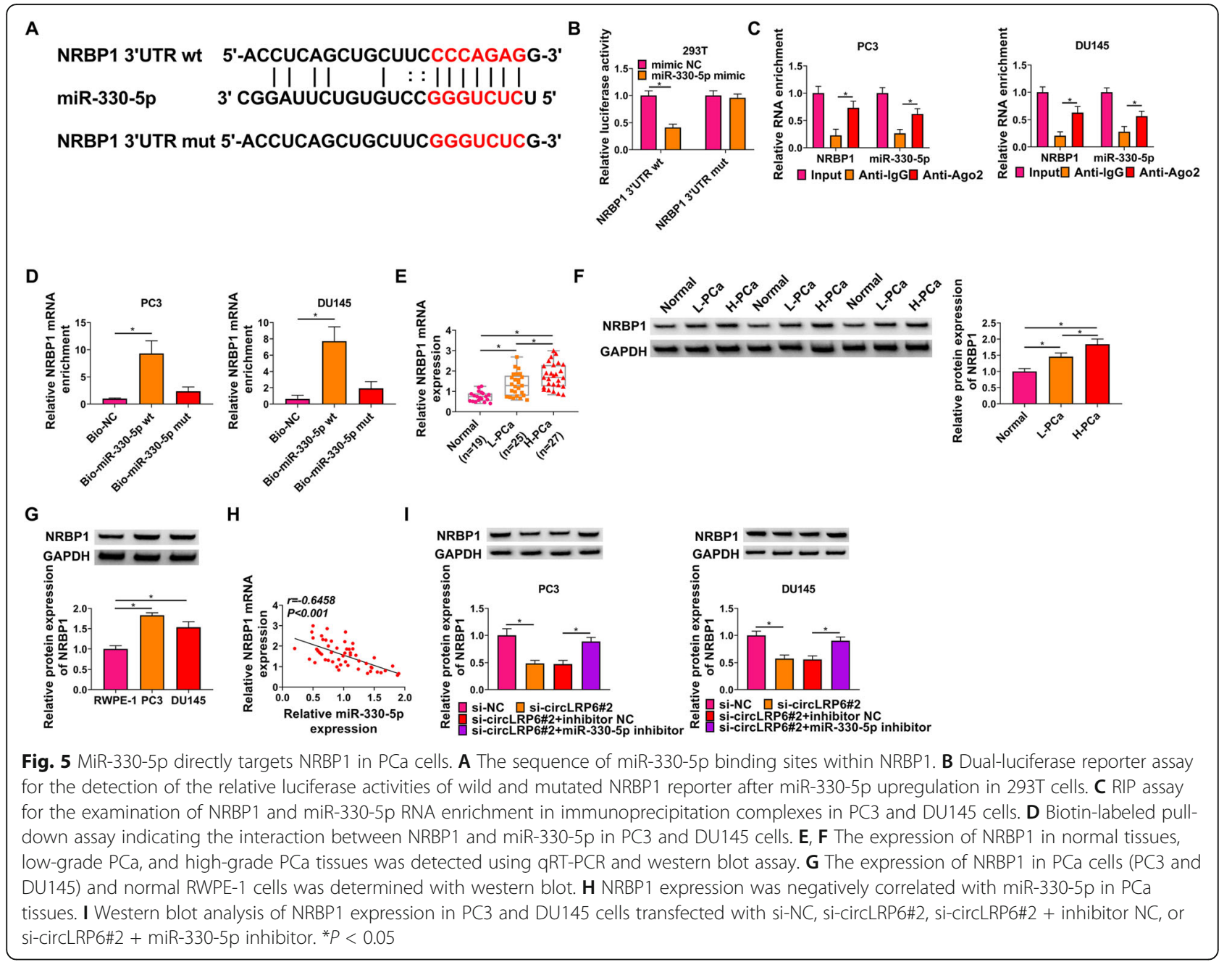

NRBP1 expression was found to be highly expressed in high-grade $\mathrm{PCa}$ tissues in comparison to the low-grade and normal PCa tissues (Fig. 5E, F), as well as in PCa cells compared with the normal RWPE-1 cells (Fig. 5G). Moreover, its expression was negatively correlated with miR330-5p in PCa tissues (Fig. 5H). Further western blot analysis suggested that circLRP6 knockdown reduced the level of NRBP1 in PC3 and DU145 cells, which was rescued by the inhibition of miR-330-5p (Fig. 5I). Thus, we confirmed that miR-330-5p targeted NRBP1 and circLRP6 could regulate NRBP1 expression by sponging miR-330$5 p$.

\section{MiR-330-5p restrains $\mathrm{PCa}$ cell growth and metastasis via targeting NRBP1}

We then investigated whether miR-330-5p mediated PCa cell growth and metastasis via NRBP1. qRT-PCR analysis showed that miR-330-5p mimics significantly elevated miR-330-5p in PC3 and DU145 cells (Fig. 6A).
Then PC3 and DU145 cells were co-transfected with miR-330-5p mimic and NRBP1 overexpressing pcDNA to perform rescue assay. Western blot analysis indicated miR-330-5p mimic reduced the expression level of NRBP1 in PC3 and DU145 cells, which was rescued by the transfection of pcDNA-NRBP1 (Fig. 6B). CCK-8, colony formation, and EDU incorporation assays were used to detect cell proliferation. Results showed that miR330-5p mimic led to the increase of the proliferation rate (Fig. 6C), colony-formation abilities (Fig. 6D), and DNA synthesis activities (Fig. 6E) in PC3 and DU145 cells, which were reversed by the overexpression of NRBP1 (Fig. 6C-E). Flow cytometry revealed that NRBP1 overexpression attenuated miR-330-5p mimic-mediated cell cycle arrest and cell apoptosis suppression in PC3 and DU145 cells (Fig. 6F, G). Furthermore, we also demonstrated that miR-330-5p mimic suppressed PC3 and DU145 cell to invade, migrate and EMT, while this condition was counteracted by NRBP1 overexpression (Fig. 


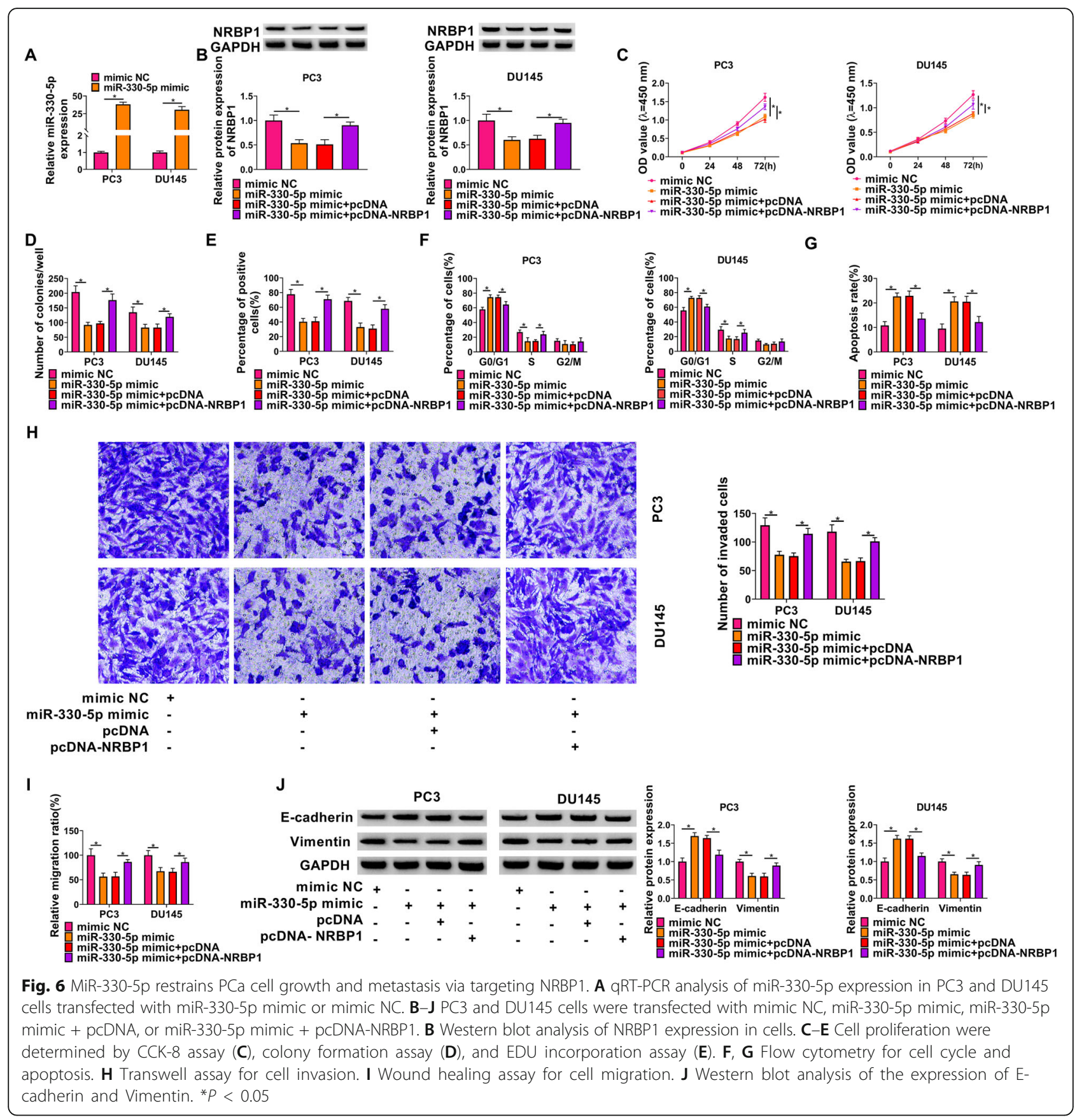

$6 \mathrm{H}-\mathrm{J})$. Collectively, miR-330-5p/NRBP1 axis was engaged in $\mathrm{PCa}$ cell growth and metastasis.

\section{CircLRP6 knockdown impedes PCa tumor growth and EMT in vivo}

Subsequently, the biological function of circLRP6 in vivo was explored using the PC3 tumor model with $\mathrm{BABL} / \mathrm{c}$ nude mice. Consistent with the results in vitro, circLRP6 silencing prominently decreased tumor volume and weight compared with those in the
sh-NC group (Fig. 7A, B). Molecular analysis showed that the expression of circLRP6 and NRBP1 was lower, while miR-330-5p expression was higher in tumors isolated from sh-circLRP6 groups (Fig. 7C, D). The results of western blot analysis suggested that circLRP6 knockdown led to an increase of E-cadherin protein level and a decrease of Vimentin protein level in tumors of mice (Fig. 7D). Furthermore, IHC assay confirmed that the expression of Ki-67 and NRBP1 was downregulated in tumors of sh-circLRP6 group 
compared with sh-NC group (Fig. 7E). These results suggested that circLRP6 knockdown suppressed PCa tumor growth and EMT in vivo.

\section{Discussion}

Recently, aberrant expression of circRNAs has been shown in $\mathrm{PCa}$, and circRNAs have been revealed to play significant roles in $\mathrm{PCa}$ progression $[19,20]$. The functions and regulation of circRNAs have attracted great research interest for discovering novel targets in the diagnosis and treatment of PCa. In the present study, through the use of circular RNA microarrays, circLRP6 was found to be highly expressed in PCa tissues. Then, the uncharacterized circLRP6 in PCa tumorigenesis was investigated. As expected, circLRP6 expression also was higher in clinical PCa tissues, especially in high-graded PCa tissues. In vitro functional studies indicated that knockdown of circLRP6 could suppress PCa cell proliferation, invasion, migration, but induce apoptosis. Ecadherin is a transmembrane glycoprotein, involved in cell-cell adhesion and EMT. Vimentin is highly expressed in mesenchymal cells and positively correlated with increased metastasis [21]. In this study, the results of western blot analysis suggested that circLRP6 silencing reduced Vimentin expression and increased Ecadherin expression. Therefore, we demonstrated that knockdown of circLRP6 suppressed PCa cell growth and metastasis in vitro. Importantly, consistent with the results in vitro, results of tumor formation assay confirmed that circLRP6 silencing prominently decreased tumor growth and EMT in vivo. All these results indicated a tumor-promoter role of circLRP6 in PCa.

Mounting evidence suggests that circRNAs can serve as endogenous sponges of miRNAs to protect the target mRNAs from repression [22, 23]. Wang et al. revealed that circITCH worked as a sponge in PCa, regulated the expression of miR-17-5p to increase HOXB13 expression level and repressed malignant phenotype of $\mathrm{PCa}$ [24]. Yang et al. showed circAMOTL1L increased Pcdha expression by sponging miR-193a-5p and suppressed migration and invasion of $\mathrm{PCa}$ cells through leading to EMT inhibition [25]. In the present study, we also
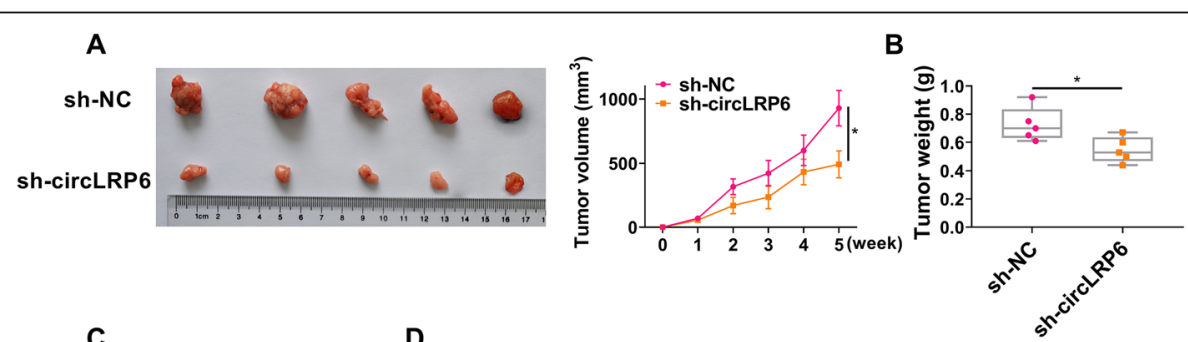

C

D
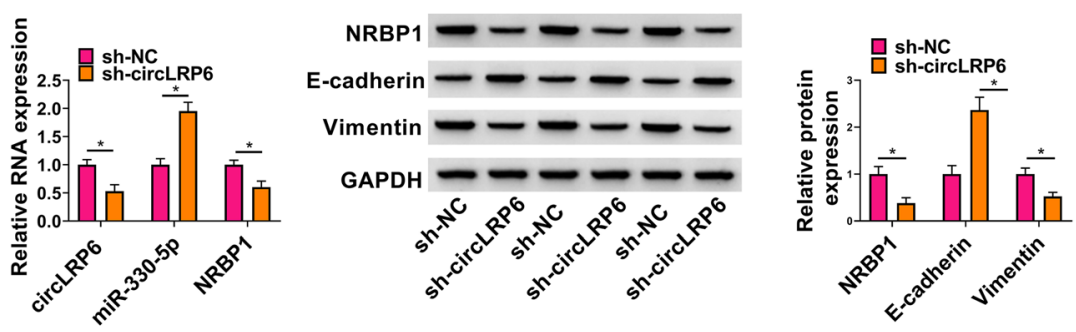

E

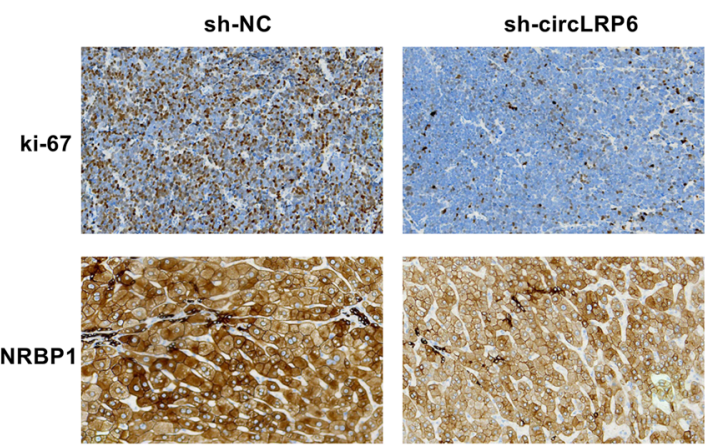

Fig. 7 CircLRP6 knockdown impedes PCa tumor growth and EMT in vivo. A subcutaneous tumor model was established. A Image of tumors (left panel) and in vivo growth curve (right panel). B Tumor weight of each group was analyzed at day 35. C qRT-PCR analysis of circLRP6, NRBP1, and miR-330-5p expression in tumors of mice from each group. D Western blot analysis of NRBP1, E-cadherin, and Vimentin protein levels in the tumor of mice from each group. $\mathbf{E}$ Representative images of $\mathrm{HC}$ staining of NRBP and Ki-67 in tumors. ${ }^{*} P<0.05$ 
confirmed that circLRP6 acted as a sponge for miR-330$5 p$ to upregulate NRBP1 expression in PCa in vitro and in vivo. However, whether circLRP6 exerted its action via miR-330-5p/NRBP1 axis remains unclear.

MiRNAs are one kind of noncoding RNAs composed of 21-23 nucleotides, which have been reported to involve in the progression of many types of cancers though modulating cellular biological processes such as cell proliferation, migration, and apoptosis [26, 27]. Current treatments for PCa mainly include surgery, chemotherapy, radiotherapy, androgen deprivation therapy, and castration [28], Egidi et al. showed that miRNAs, including miR-21 and miR-141, could be implicated in postsurgical inflammatory processes and did not impacted by radical prostatectomy [29]. Besides that, miRNAs, in serum or urine exfoliated cell, were demonstrated to be reliable invasive biomarkers for the diagnosis and prognosis of PCa $[28,30]$. Moreover, they were also discovered to be stable in post-digital rectal examination (DRE) urine sediments and might be used as reference genes for the assessment of suspected $\mathrm{PCa}$ [31]. In the meanwhile, numerous miRNAs are revealed to participate in the tumorigenesis of $\mathrm{PCa}$ [32]. Therefore, abnormalities of miRNAs expression may be promising biomarkers for the prediction, diagnosis, and treatment in $\mathrm{PCa}$.

MiR-330-5p has been proven to be connected with diverse cancers. However, the function of miR-330-5p is complicated because it can be a tumor suppressor or a carcinogen in the context of different cancers. MiR-330$5 p$ is identified as tumor suppressor in osteosarcoma by suppressing cell growth and invasion through the inhibition of the proto-oncogene survivin [33]. In glioblastoma, miR-330-5p suppressed ITGA5 expression to impede cell proliferation and invasion [34]. Besides that, miR-330-5p repressed cell invasive phenotype through downregulating MMP1 expression in esophageal adenocarcinoma [35]. Additionally, miR-330-5p was demonstrated to function as onco-miR in cervical cancer by enhancing cancer cell invasion and metastasis [36]. Moreover, miR-330-5p was confirmed to suppress SPRY2 to promote proliferation via MAPK/ERK signaling in hepatocellular carcinoma [37]. In PCa, miR-330$5 \mathrm{p}$ was demonstrated to restrain $\mathrm{PCa}$ growth and metastasis through the LEF1-AS1/miR-330-5p/LEF1 pathway in vitro and in vivo [38]. All these findings suggested the involvement of miR-330-5p in cancer growth and metastasis.

MiRNA can regulate gene expression via binding to the 3'UTR of the target mRNA at the level of mRNA turnover or translation, thereby exerting their effects in carcinogenesis [27, 39, 40]. NRBP1 is a ubiquitously expressed, highly conserved pseudokinase [41]. NRBP1 has been revealed to exert different functions in different cancers $[42,43]$. In PCa, high NRBP1 expression could be of prognostic value in $\mathrm{PCa}$ patients, and enhanced cancer cell proliferation [44]. Besides, Yan et al. showed NRBP1 was involved in miR-519d-induced inhibition of $\mathrm{PCa}$ cell growth and invasion [45]. Thus, NRBP1 is considered to be an oncogene in PCa. In the current study, a mutual antagonism between miR-330-5p and circLRP6 on affecting PCa cell growth and metastasis was investigated. Importantly, we also demonstrated that miR-330-5p overexpression suppressed the progression of PCa, which was attenuated by NRBP1 upregulation. Taken together, a functional regulatory network in $\mathrm{PCa}$ growth and metastasis was identified.

\section{Conclusion}

In conclusion, our findings suggested that circLRP6 increased NRBP1 expression level by miR-330-5p, subsequent promoted PCa growth and metastasis, suggesting a new direction for PCa treatment.

\section{Supplementary Information}

The online version contains supplementary material available at https://doi. org/10.1186/s12957-021-02287-2.

Additional file 1: Figure S1. The effects of circLRP6/miR-330-5p axis on normal RWPE-1 cells. (A-D) RWPE-1 cells were transfected with si-NC, sicircLRP6\#2, si-circLRP6\#2 + inhibitor NC, or si-circLRP6\#2 + miR-330-5p inhibitor. (A) qRT-PCR of miR-330-5p expression in cells. (B) Cell proliferation were determined by colony formation assay. (C) Flow cytometry for cell apoptosis. (D) Transwell assay for cell invasion. ${ }^{*} P<0.05$.

Additional file 2: Figure S2. The effects of circLRP6 or miR-330-5p on the expression levels of potential target genes. (A) qRT-PCR of miR-1247, miR-153, miR-198, miR-326, miR-515-5p, miR-543, and miR-330-5p expression levels in PCa cells (PC3 and DU145) and normal RWPE-1 cells. (B, C) qRT-PCR of miR-198, miR-326, miR-515-5p, miR-543, and miR-330-5p expression levels in PC3 and DU145 cells transfected with si-NC or si-

circLRP6\#2. (D) Western blot analysis of the protein levels of IGF1R, MYO6, SMAD3, CCND1, NRBP1 in PC3 and DU145 cells transfected with mimic NC or miR-330-5p mimic. ${ }^{*} P<0.05$.

\section{Authors' contributions}

LQ performed experiments, analyzed data, and wrote the manuscript. CL designed research and performed experiments. XS collected the data. FZ edited the manuscript. All authors read and approved the final manuscript.

\section{Funding}

There is no funding to report.

\section{Availability of data and materials}

The data sets used and/or analyzed during the current study are available from the corresponding author on reasonable request.

\section{Declarations}

Ethics approval and consent to participate

This work was endorsed and supervised by the Ethics Committee of the Xiangyang Central Hospital (approval number 2019rs06) and was carried out according to the guidelines of Declaration of Helsinki. The procedures of this animal study were in accordance with the regulations of the Ethics

Committee of Xiangyang Central Hospital (approval number AN2019S011) and performed in accordance with the guidelines of the National Animal

Care and Ethics Institution. 


\section{Consent for publication}

Not applicable

\section{Competing interests}

The authors declare that they have no competing interests.

\section{Received: 13 April 2021 Accepted: 4 June 2021}

\section{Published online: 22 June 2021}

\section{References}

1. Torre LA, Bray F, Siegel RL, Ferlay J, Lortet-Tieulent J, Jemal A. Global cancer statistics, 2012. CA Cancer J Clin. 2015;65(2):87-108. https://doi.org/10.3322/ caac.21262.

2. Pernar $\mathrm{CH}$, Ebot EM, Wilson KM, Mucci LA. The epidemiology of prostate cancer. Cold Spring Harb Perspect Med. 2018;8(12):a030361. https://doi. org/10.1101/cshperspect.a030361.

3. Gaztañaga M, Crook J. Androgen deprivation therapy: minimizing exposure and mitigating side effects. J Natl Compr Canc Netw. 2012;10(9):1088-95. https://doi.org/10.6004/jnccn.2012.0115.

4. Karantanos T, Corn PG, Thompson TC. Prostate cancer progression after androgen deprivation therapy: mechanisms of castrate resistance and novel therapeutic approaches. Oncogene. 2013;32(49):5501-11. https://doi.org/1 0.1038/onc.2013.206

5. Wang JJ, Shan K, Liu BH, Liu C, Zhou RM, Li XM, et al. Targeting circular RNA-ZRANB1 for therapeutic intervention in retinal neurodegeneration. Cell Death Dis. 2018:9(5):540. https://doi.org/10.1038/s41419-018-0597-7.

6. Santosh B, Varshney A, Yadava PK. Non-coding RNAs: biological functions and applications. Cell Biochem Funct. 2015;33(1):14-22. https://doi.org/10.1 002/cbf.3079.

7. $Y u$ T, Wang $Y$, Fan $Y$, Fang $N$, Wang $T$, Xu T, et al. CircRNAs in cancer metabolism: a review. J Hematol Oncol. 2019;12(1):90. https://doi.org/10.11 86/s13045-019-0776-8

8. Bach DH, Lee SK, Sood AK. Circular RNAs in cancer. Mol Ther Nucleic Acids. 2019;16:118-29. https://doi.org/10.1016/j.omtn.2019.02.005.

9. Salzman J, Chen RE, Olsen MN, Wang PL, Brown PO. Cell-type specific features of circular RNA expression. PLoS Genet. 2013;9(9):e1003777. https:// doi.org/10.1371/journal.pgen.1003777.

10. Zhang HD, Jiang LH, Sun DW, Hou JC, Ji ZL. CircRNA: a novel type of biomarker for cancer. Breast Cancer. 2018;25(1):1-7. https://doi.org/10.1007/ s12282-017-0793-9.

11. Verduci L, Strano S, Yarden Y, Blandino G. The circRNA-microRNA code: emerging implications for cancer diagnosis and treatment. Mol Oncol. 2019; 13(4):669-80. https://doi.org/10.1002/1878-0261.12468.

12. Shen Z, Zhou L, Zhang C, Xu J. Reduction of circular RNA Foxo3 promotes prostate cancer progression and chemoresistance to docetaxel. Cancer Lett. 2020;468:88-101. https://doi.org/10.1016/j.canlet.2019.10.006.

13. Xiang Z, Xu C, Wu G, Liu B, Wu D. CircRNA-UCK2 increased TET1 inhibits proliferation and invasion of prostate cancer cells via sponge MiRNA-767-5p. Open Med (Wars). 2019;14:833-42. https://doi.org/10.1515/med-2019-0097.

14. Li T, Sun X, Chen L. Exosome circ 0044516 promotes prostate cancer cell proliferation and metastasis as a potential biomarker. J Cell Biochem. 2020; 121(3):2118-26. https://doi.org/10.1002/jcb.28239.

15. Zheng S, Qian Z, Jiang F, Ge D, Tang J, Chen H, et al. CircRNA LRP6 promotes the development of osteosarcoma via negatively regulating KLF2 and APC levels. Am J Transl Res. 2019;11(7):4126-38.

16. Xue J, Chen C, Luo F, Pan X, Xu H, Yang P, et al. CircLRP6 regulation of ZEB1 via miR-455 is involved in the epithelial-mesenchymal transition during arsenite-induced malignant transformation of human keratinocytes. Toxicol Sci. 2018:162(2):450-61. https://doi.org/10.1093/toxsci/kfx269.

17. Wang J, Zhu W, Tao G, Wang W. Circular RNA circ-LRP6 facilitates Mycdriven tumorigenesis in esophageal squamous cell cancer. Bioengineered. 2020;11(1):932-8. https://doi.org/10.1080/21655979.2020.1809922.

18. Li T, Gao X, Han L, Yu J, Li H. Identification of hub genes with prognostic values in gastric cancer by bioinformatics analysis. World J Surg Oncol. 2018; 16(1):114. https://doi.org/10.1186/s12957-018-1409-3

19. Greene J, Baird AM, Casey O, Brady L, Blackshields G, Lim M, et al. Circular RNAs are differentially expressed in prostate cancer and are potentially associated with resistance to enzalutamide. Sci Rep. 2019:9(1):10739. https:// doi.org/10.1038/s41598-019-47189-2.

20. Yan Z, Xiao Y, Chen Y, Luo G. Screening and identification of epithelialto-mesenchymal transition-related circRNA and miRNA in prostate cancer. Pathol Res Pract. 2020;216(2):152784. https://doi.org/10.1016/j. prp.2019.152784.

21. Nijkamp MM, Span PN, Hoogsteen IJ, van der Kogel AJ, Kaanders JHAM, Bussink J. Expression of E-cadherin and vimentin correlates with metastasis formation in head and neck squamous cell carcinoma patients. Radiother Oncol. 2011;99(3):344-8. https://doi.org/10.1016/j.radonc.2011.05.066.

22. Hansen TB, Jensen TI, Clausen BH, Bramsen JB, Finsen B, Damgaard CK, et al. Natural RNA circles function as efficient microRNA sponges. Nature. 2013; 495(7441):384-8. https://doi.org/10.1038/nature11993.

23. Thomson DW, Dinger ME. Endogenous microRNA sponges: evidence and controversy. Nat Rev Genet. 2016;17(5):272-83. https://doi.org/10.1 038/nrg.2016.20.

24. Wang X, Wang R, Wu Z, Bai P. Circular RNA ITCH suppressed prostate cancer progression by increasing HOXB13 expression via spongy miR-17-5p. Cancer Cell Int. 2019;19(1):328. https://doi.org/10.1186/s12935-019-0994-8.

25. Yang Z, Qu CB, Zhang Y, Zhang WF, Wang DD, Gao CC, et al. Dysregulation of p53-RBM25-mediated circAMOTL1L biogenesis contributes to prostate cancer progression through the circAMOTL1LmiR-193a-5p-Pcdha pathway. Oncogene. 2019;38(14):2516-32. https://doi. org/10.1038/s41388-018-0602-8

26. $\mathrm{Xu} \mathrm{L}$, Zheng $\mathrm{Q}$. Identification and validation of a miRNA-related expression signature for tumor mutational burden in colorectal cancer. World J Surg Oncol. 2021;19(1):56. https://doi.org/10.1186/s12957-021-02137-1.

27. Cai R, Lu Q, Wang D. Construction and prognostic analysis of miRNA-mRNA regulatory network in liver metastasis from colorectal cancer. World I Surg Oncol. 2021;19(1):7. https://doi.org/10.1186/s12957-020-02107-z.

28. Guelfi G, Cochetti G, Stefanetti V, Zampini D, Diverio S, Boni A, et al. Next Generation Sequencing of urine exfoliated cells: an approach of prostate cancer microRNAs research. Sci Rep. 2018;8(1):7111. https://doi.org/10.1038/ s41598-018-24236-y.

29. Egidi MG, Cochetti G, Serva MR, Guelfi G, Zampini D, Mechelli L, et al. Circulating microRNAs and kallikreins before and after radical prostatectomy: are they really prostate cancer markers? Biomed Res Int. 2013:2013:241780.

30. Cochetti G, Poli G, Guelfi G, Boni A, Egidi MG, Mearini E. Different levels of serum microRNAs in prostate cancer and benign prostatic hyperplasia: evaluation of potential diagnostic and prognostic role. Onco Targets Ther. 2016:9:7545-53. https://doi.org/10.2147/OTT.S119027.

31. Egidi MG, Cochetti G, Guelfi G, Zampini D, Diverio S, Poli G, et al. Stability assessment of candidate reference genes in urine sediment of prostate cancer patients for miRNA applications. Dis Markers. 2015;2015:973597.

32. Cochetti G, Rossi de Vermandois JA, Maulà V, Giulietti M, Cecati M, Del Zingaro $M$, et al. Role of miRNAs in prostate cancer: do we really know everything? Urol Oncol. 2020;38(7):623-35. https://doi.org/10.1016/j. urolonc.2020.03.007.

33. Wang H, Liu L, Fang S. MicroRNA-330-5p inhibits osteosarcoma cell growth and invasion by targeting the proto-oncogene survivin. Mol Med Rep. 2019; 20(3):2236-44. https://doi.org/10.3892/mmr.2019.10447.

34. Feng L, Ma J, Ji H, Liu Y, Hu W. miR-330-5p suppresses glioblastoma cell proliferation and invasiveness through targeting ITGA5. Biosci Rep. 2017; 37(3):BSR20170019.

35. Bibby BAS, Miranda CS, Reynolds JV, Cawthorne CJ, Maher SG. Silencing microRNA-330-5p increases MMP1 expression and promotes an invasive phenotype in oesophageal adenocarcinoma. BMC Cancer. 2019;19(1):784. https://doi.org/10.1186/s12885-019-5996-3.

36. Chen S, Wang J. HAND2-AS1 inhibits invasion and metastasis of cervical cancer cells via microRNA-330-5p-mediated LDOC1. Cancer Cell Int. 2019; 19(1):353. https://doi.org/10.1186/s12935-019-1048-y.

37. Xiao $S$, Yang $M$, Yang $H$, Chang $R$, Fang F, Yang L. miR-330-5p targets SPRY2 to promote hepatocellular carcinoma progression via MAPK/ERK signaling. Oncogenesis. 2018;7(11):90.

38. Liu DC, Song LL, Liang Q, Hao L, Zhang ZG, Han CH. Long noncoding RNA LEF1-AS1 silencing suppresses the initiation and development of prostate cancer by acting as a molecular sponge of miR-330-5p via LEF1 repression. J Cell Physiol. 2019;234(8):12727-44. https://doi.org/10.1002/jcp.27893.

39. Zhang C, Bo C, Guo L, Yu P, Miao S, Gu X. BCL2 and hsa-miR-181a-5p are potential biomarkers associated with papillary thyroid cancer based on bioinformatics analysis. World J Surg Oncol. 2019;17(1):221. https://doi.org/1 0.1186/s12957-019-1755-9.

40. Liao G, Tang J, Wang D, Zuo H, Zhang Q, Liu Y, et al. Selenium nanoparticles (SeNPs) have potent antitumor activity against prostate 
cancer cells through the upregulation of miR-16. World J Surg Oncol. 2020; 18(1):81. https://doi.org/10.1186/s12957-020-01850-7.

41. Kerr JS, Wilson CH. Nuclear receptor-binding protein 1: a novel tumour suppressor and pseudokinase. Biochem Soc Trans. 2013:41(4):1055-60. https://doi.org/10.1042/BST20130069.

42. Wilson $\mathrm{CH}$, Crombie $\mathrm{C}$, van der Weyden L, Poulogiannis G, Rust AG, Pardo $M$, et al. Nuclear receptor binding protein 1 regulates intestinal progenitor cell homeostasis and tumour formation. EMBO J. 2012;31(11):2486-97. https://doi.org/10.1038/emboj.2012.91

43. Liao Y, Yang Z, Huang J, Chen H, Xiang J, Li S, et al. Nuclear receptor binding protein 1 correlates with better prognosis and induces caspasedependent intrinsic apoptosis through the JNK signalling pathway in colorectal cancer. Cell Death Dis. 2018;9(4):436. https://doi.org/10.1038/ s41419-018-0402-7.

44. Ruiz C, Oeggerli M, Germann M, Gluderer S, Stocker H, Andreozzi M, et al. High NRBP1 expression in prostate cancer is linked with poor clinical outcomes and increased cancer cell growth. Prostate. 2012;72(15):1678-87. https://doi.org/10.1002/pros.22521.

45. Yan CQ, Lu YH, Tang SM, Fan WX. MiR-519d inhibits prostate cancer cell proliferation, cycle and invasion via targeting NRBP1. Eur Rev Med Pharmacol Sci. 2018;22(10):2985-90. https://doi.org/10.26355/eurrev_201 805 15054.

\section{Publisher's Note}

Springer Nature remains neutral with regard to jurisdictional claims in published maps and institutional affiliations.

Ready to submit your research? Choose BMC and benefit from:

- fast, convenient online submission

- thorough peer review by experienced researchers in your field

- rapid publication on acceptance

- support for research data, including large and complex data types

- gold Open Access which fosters wider collaboration and increased citations

- maximum visibility for your research: over $100 \mathrm{M}$ website views per year

At $\mathrm{BMC}$, research is always in progress.

Learn more biomedcentral.com/submissions 